\title{
Amino Acids in Cerebrospinal and Brain Interstitial Fluid in Experimental Pneumococcal Meningitis
}

\author{
LUIS GUERRA-ROMERO, JAY H. TUREEN, MIKE A. FOURNIER, VICTORIA MAKRIDES, AND \\ MARTIN G. TÄUBER \\ The Infectious Diseases Laboratories, [L.G.-R., J.H.T., M.A.F., M.G.T.], and the Laboratory for Clinical \\ Pharmacology [V.M.], San Francisco General Hospital, and the Departments of Medicine [L.G.-R., M.A.F., \\ V.M., M.G.T.], and Pediatrics [J.H.T.], University of California, San Francisco, California 94143
}

\begin{abstract}
Excitatory amino acids are increasingly implicated in the pathogenesis of neuronal injury induced by a variety of CNS insults, such as ischemia, trauma, hypoglycemia, and epilepsy. Little is known about the role of amino acids in causing CNS injury in bacterial meningitis. Several amino acids were measured in cerebrospinal fluid and in microdialysis samples from the interstitial fluid of the frontal cortex in a rabbit model of pneumoccocal meningitis. Cerbrospinal fluid concentrations of glutamate, aspartate, glycine, taurine, and alanine increased significantly in infected animals. Among the amino acids with known excitatory or inhibitory function, interstitial fluid concentrations of glutamate were significantly elevated (by $470 \%$ ). Alanine, a marker for anaerobic glycolysis, also increased in the cortex of infected rabbits. The elevated glutamate concentrations in the brain extracellular space suggest that excitotoxic neuronal injury may play a role in bacterial meningitis. (Pediatr Res 33: 510-513, 1993)
\end{abstract}

\section{Abbreviations}

CSF, cerebrospinal fluid

EAA, excitatory amino acid

cfu, colony-forming unit

EAA, physiologic neurotransmitters in the mammalian CNS, are increasingly implicated in the pathogenesis of neuronal injury induced by a variety of CNS insults, such as global and focal ischemia, brain and spinal cord trauma, hypoglycemia, and epilepsy (1-7). There is good evidence (8) that increased interstitial concentrations of EAA produce neuronal damage and death by mechanisms that appear to involve influx of calcium into neurons through receptor-gated ion channels.

Neurologic sequelae develop in up to $30 \%$ of survivors of bacterial meningitis and include hearing loss, learning disabilities, focal deficits, and hydrocephalus $(9,10)$. The pathophysiology of bacterial meningitis is characterized by several complex events, including cerebral metabolic disturbances, brain edema, intracranial hypertension, and alterations of cerebral blood flow $(11,12)$. The molecular mechanisms of brain injury remain

Received July 31, 1992; accepted December 18, 1992.

Correspondence: Martin G. Täuber, M.D., Infectious Diseases Laboratories, Box 0811, University of California San Francisco, 3rd and Parnassus Ave., San Francisco, CA 94143 .

Supported in part by grants from the Ministerio de Education i Licencia and the Sociedad de Enfermendades Infectiosas i Microbiologia Clincia of Spain (L.G.R.) and from Roche Laboratories, Nutley, NY (M.G.T.) and PHS Grant NS27310 (J.H.T.). poorly understood. Cerebral blood flow studies in humans and experimental animals with bacterial meningitis suggest that, after an initial hyperemic phase (13), ischemia occurs under certain circumstances and may contribute to the development of neuronal injury (14-16). As with other forms of ischemia-induced CNS injury it is conceivable that the harmful effects of ischemia could be potentiated by EAA during meningitis.

A few studies have examined concentrations of amino acids in CSF in bacterial meningitis. A first report in 1975 found increased levels of glutamic acid, glutamine, and $\gamma$-aminobutyric acid in the CSF of patients with bacterial meningitis (17). Other authors have also reported abnormal CSF concentrations of amino acids, but the importance of these changes has not been explored in detail (18-20). Importantly, it is not known whether amino acid concentrations in the CSF reflect changes occurring in the brain interstitial fluid, the medium relevant for the potential neurotoxicity of EAA. To test the hypothesis that changes in amino acids may play a role in CNS injury during meningitis, we measured in the present study brain interstitial fluid concentrations of several amino acids in a rabbit model of pneumoccocal meningitis and determined whether changes similar to those observed in the CSF during meningitis occur in the brain. Interstitial fluid concentrations of amino acids were measured in the cortex because of its proximity to the inflammation in the subarachnoid space and because pathologic changes were primarily found in the cortex in a recent histopathologic study in rats with experimental pneumococcal meningitis (21).

\section{MATERIALS AND METHODS}

Model of experimental meningitis. Meningitis was produced by intracisternal injection of $0.3 \mathrm{~mL}$ of saline containing an inoculum of $1 \times 10^{6}$ to $1 \times 10^{7} \mathrm{cfu}$ of Streptococcus pneumoniae, type 3, as described by Dacey and Sande (22). These studies were approved by the Committee on Animal Research of the University of California San Francisco and followed the NIH Guidelines for the Care and Use of Laboratory Animals. New Zealand White rabbits $(2.0$ to $2.5 \mathrm{~kg})$ were anesthetized by i.v. urethane $(2.0 \mathrm{~g} /$ $\mathrm{kg}$ i.v.) for all procedures. Anesthesia was maintained by supplemental doses of urethane $(0.5 \mathrm{~g} / \mathrm{kg}$ i.v. $)$. A polyethylene catheter (PE-90) was inserted into the femoral artery for measurement of blood pressure and blood sampling. A dental acrylic cap containing a turnbuckle was affixed to the outer table of cranial bone by steel screws, which allowed immobilization of the animals in a restraining frame. The cisterna magna was punctured with a 25-gauge, 3.5-inch spinal needle (Becton Dickinson \& Co., Franklin Lake, NJ) for infecting animals and for sampling CSF.

Microdialysis probes (2-mm membrane tip length, bioanalytical Systems, West Lafayette, IN) were tested before implantation by perfusion with sterile, artificial CSF (composition: $0.75 \%$ 
$\mathrm{NaCl}, 0.02 \% \mathrm{KCl}, 0.015 \% \mathrm{CaCl}_{2}$, and $0.019 \% \mathrm{MgCl}_{2}$ in filtersterilized distilled water) while suspended in a standard solution of glutamate and lactate. Concentrations of the two molecules in the effluent were compared with that in the standard solution to assess the recovery rate for each probe. Extraction rates for glutamate and lactate were very similar, and probes used in the study had extraction rates for glutamate between 7 and $37 \%$.

Probes were implanted in the left frontal cortex (coordinates: $4.0 \mathrm{~mm}$ anterior to the bregma, $4.0 \mathrm{~mm}$ lateral to the sagittal suture, and $3.0 \mathrm{~mm}$ from the pial surface) using a micromanipulator. After placement, the probes were affixed to the skull with dental cement. Throughout the study, probes were perfused with artificial CSF with use of a syringe pump (model 931, Harvard Apparatus, Millis, MA) at a rate of $1.36 \mu \mathrm{L} / \mathrm{min}$. At the conclusion of the study, rabbits were killed with a lethal dose of pentobarbital $(150 \mathrm{mg} / \mathrm{kg}$ i.v.), and a postmortem microdialysis sample was collected to document the continued functioning of the probe as evidenced by a massive increase in glutamate concentrations. Evans blue dye was then pumped through the probes to allow macroscopic confirmation of the correct anatomical placement of the probes after removal of the brain.

Experimental protocol. After placement of the animals in the frames and probe implantation, $0.3 \mathrm{~mL}$ of the pneumococcal inoculum $(n=21)$ or $0.3 \mathrm{~mL}$ of sterile, nonbacteriostatic saline (controls, $n=10$ ) were administered intracisternally. Blood samples for arterial lactate concentrations and for blood gases were sampled at 0,16 , and $22 \mathrm{~h}$ and CSF samples $(0.2 \mathrm{~mL})$ were obtained for amino acid concentrations, lactate concentrations, bacterial titers, and white blood cell counts at 0 and $22 \mathrm{~h}$. Blood pressure was measured at $22 \mathrm{~h}$ before killing the animals. The 22-h time point was chosen as end point in this study because, in our model, meningitis is fully developed by 20 to $24 \mathrm{~h}$ with maximal CSF bacterial titers and leukocytosis, but animals are not yet agonal and do not have seizures $(15,16)$. Fractions of microdialysate (sampled during 1 - to 2 -h intervals) were collected in polypropylene tubes for analysis throughout the experiment and are reported as baseline and peak values derived from the first and last two samples. The sample obtained during the $1 \mathrm{st} h$ after implantation was discarded because of the known effect of implantation injury on glutamate concentrations. CSF samples were centrifuged after collection at $8000 \times g$ for 5 min to remove cellular elements, and CSF and microdialysis samples were then frozen and kept at $-70^{\circ} \mathrm{C}$ until analyzed. All amino acid concentrations remained stable in the biologic samples when kept at $-70^{\circ} \mathrm{C}$ for $2 \mathrm{mo}$, and samples were processed within that time period, with the exception of aspartate, which consistently increased during storage approximately 2 -fold.

Experimental parameters. Bacterial titers in CSF were determined by serial 10 -fold dilution of CSF in saline, after overnight incubation on blood agar plates at $37^{\circ} \mathrm{C}$ in $5 \% \mathrm{CO}_{2}$, and expressed as $\log _{10} \mathrm{cfu} / \mathrm{mL}$. White blood cell counts in CSF were determined in a Neubaur hemocytometer. CSF and blood concentrations of lactate were measured immediately after sampling in a twochannel autoanalyzer (YSI $2300 \mathrm{G} / \mathrm{L}$, Yellow Springs Instrument Co., Yellow Springs, OH). Glutamate, aspartate, asparagine, serine, glycine, threonine, taurine, and alanine were measured in the CSF and microdialysate by the following technique: amino acids were derivatized with ortho-phtaldialdehyde and t-butyl thiol, and then separated by HPLC (chromatograph HP 1090, Hewlett Packard, Santa Clara, CA), using a Rainin, 3- $\mu \mathrm{m}$ Microsorb C18 reverse-phase analytical column (80-200-C3, Rainin Instrument Co., Woburg, MA) with a flow rate of $1.0 \mathrm{~mL} / \mathrm{min}$ and a running time of $40 \mathrm{~min}$. Mobile phase A consisted of $1 \%$ tetrahydrofuran and $19 \%$ methanol in $0.05 \mathrm{M}$ sodium acetate buffer, $\mathrm{pH} 5.9$, and mobile phase $\mathrm{B}$ consisted of $80 \%$ methanol in $0.05 \mathrm{M}$ sodium acetate, $\mathrm{pH} 5.9$. Amino acids were detected fluorometrically (detector HP 1046A, Hewlett Packard). Not all peaks were mathematically analyzed. Microdialysis concentrations of glutamate and serine (as a control amino acid without neurotransmitter function) were analyzed in all animals; aspar- agine, glutamine, glycine, and threonine were analyzed in consecutive animals 6 to 21 , and taurine and alanine were analyzed in consecutive animals 6 to 14. These two subgroups of animals were representative for the whole group based on the glutamate concentrations in microdialysis fluid and on CSF parameters of lactate, glucose, and bacterial titer. CSF glutamate concentrations were determined for animals 1 to 8 and 16 to 21 , whereas the other CSF amino acids were analyzed in animals $8,10,11,15$, 16 , and 18 to 21 . These samples were chosen randomly, and no obtained values were excluded from analysis.

Arterial blood gases were measured in a blood gas analyzer (Radiometer ABL 2, Radiometer A/S, Copenhagen, Denmark). Arterial pressure was measured with a water-filled pressure transducer (model P-23 XL, Gould Inc., Santa Clara, CA) and recorded on a multichannel polygraph $(5 / 6 \mathrm{H}$ Recorder, Gilson Medical Electronics, Middleton, WI).

Statistical analysis. Data are expressed as mean \pm SD. Lactate concentrations in CSF or blood, blood pressure, $\mathrm{pH}, \mathrm{HCO}_{3}$, and $\mathrm{PCO}_{2}$ were analyzed by unpaired $t$ tests for comparison between groups, by paired $t$ test for comparison between first and last sample in the same group, and by linear regression analysis for correlations between parameters. Amino acid concentrations in CSF or microdialysate were analyzed by Wilcoxon signed-rank test for comparison within the same group, by Mann-Whitney rank-sum test for comparison between groups, and by linear regression analysis for correlations with other parameters.

\section{RESULTS}

Characterization of experimental meningitis. We have used a subset of the experimental animals reported in the present study to examine lactate and glucose concentrations in serum, CSF, and brain interstitial fluid during experimental pneumococcal meningitis, and these results have been recently published (23). Similar to the results in that report, infected animals in the present study had high bacterial titers in the CSF $\left(7.7 \pm 1.1 \log _{10}\right.$ $\mathrm{cfu} / \mathrm{mL}$ ) and a pronounced CSF pleocytosis at $22 \mathrm{~h}$ of infection, whereas control animals had sterile CSF cultures and normal CSF leukocyte counts (23). No significant differences in mean arterial blood pressure were observed at the end of the experiment between meningitis and control groups $(80.4 \pm 10.4$ versus 73.3 $\pm 14.9 \mathrm{~mm} \mathrm{Hg}$ ), whereas arterial blood gases demonstrated a mild metabolic acidosis in both infected and control animals that was partially compensated by a respiratory alkalosis in the infected animals but not the control animals (23). Lactate concentrations increased significantly in microdialysis fluid, CSF, and serum during development of infection, whereas they remained unchanged in control animals (23).

CSF amino acid concentrations. Amino acid concentrations in the CSF were similar in the two groups at baseline and did not show any significant changes between baseline and $22 \mathrm{~h}$ in the control animals. In contrast, CSF concentrations of glutamate, aspartate, glycine, taurine, and alanine increased significantly at $22 \mathrm{~h}$ in infected animals (Table 1). Glutamate increased 10.7-fold compared with the baseline level, and the other amino acids had increases between 42.9- (taurine) and 4.4- (alanine) fold compared with their baseline level. Asparagine, serine, and threonine did not change compared with baseline levels (Table 1). The increase in amino acids in the CSF during infection did not appear to be the result of release from multiplying pneumococci in the CSF, inasmuch as in vitro growth of the organism in CSF did not lead to a significant increase in any of the amino acids (data not shown). At $22 \mathrm{~h}$ of infection, the only pairwise correlation between absolute concentrations of amino acids in CSF that reached statistical significance was observed for the two EAA glutamate and aspartate $(r=0.92, p<0.005)$.

Microdialysate amino acids concentrations. Absolute concentrations of the amino acids measured in the microdialysis fluid are shown in Table 2. Glutamate was the only EAA elevated in animals with meningitis at $22 \mathrm{~h}(p<0.05$, Table 2$)$. When 
Table 1. CSF concentrations ( $\mu M$ ) of amino acids in rabbits with pneumococcal meningitis

\begin{tabular}{lrcc}
\hline & $\begin{array}{c}\text { Time } \\
(\mathrm{h})\end{array}$ & Mean & SD \\
\hline Glutamate $(n=14)^{*}$ & 0 & 7.89 & 3.96 \\
Aspartate $(n=8)$ & 22 & $84.42 \dagger$ & 97.45 \\
Asparagine $(n=9)$ & 0 & 2.94 & 2.14 \\
Serine $(n=9)$ & 22 & $17.73 \ddagger$ & 23.4 \\
& 0 & 8.90 & 1.72 \\
Glycine $(n=8)$ & 22 & 12.14 & 5.50 \\
Threonine $(n=8)$ & 0 & 51.07 & 12.22 \\
Taurine $(n=9)$ & 22 & 70.57 & 22.16 \\
& 0 & 13.39 & 5.19 \\
Alanine $(n=9)$ & 22 & $249.3 \ddagger$ & 349.89 \\
& 0 & 75.25 & 26.95 \\
& 22 & 120.15 & 45.64 \\
& 0 & 4.86 & 1.17 \\
& 22 & $208.5 \ddagger$ & 345.61 \\
& 0 & 30.63 & 5.75 \\
\end{tabular}

${ }^{*} n$, number of animals.

$\dagger p<0.01$ (Wilcoxon signed-ranked test).

$\ddagger p<0.05$ (Wilcoxon signed-ranked test).

Table 2. Microdialysate concentrations ( $\mu M$ ) of amino acids in frontal cortex of rabbits with pneumococcal meningitis and controls

\begin{tabular}{|c|c|c|c|c|c|}
\hline & & \multicolumn{2}{|c|}{$0 \mathrm{~h}$} & \multicolumn{2}{|c|}{$22 \mathrm{~h}$} \\
\hline & & Mean & SD & Mean & $\mathrm{SD}$ \\
\hline \multirow[t]{2}{*}{ Glutamate } & Meningitis $(n=21)^{*}$ & 0.82 & 0.47 & $2.9 \dagger$ & 4.9 \\
\hline & Control $(n=9)$ & 0.50 & 0.55 & 0.36 & 0.28 \\
\hline \multirow[t]{2}{*}{ Aspartate } & Meningitis $(n=16)$ & 0.30 & 0.21 & 0.47 & 0.46 \\
\hline & Control $(n=7)$ & 0.13 & 0.14 & 0.27 & 0.45 \\
\hline \multirow[t]{2}{*}{ Asparagine } & Meningitis $(n=16)$ & 0.43 & 0.20 & 0.48 & 0.16 \\
\hline & Control $(n=6)$ & 0.36 & 0.20 & 0.34 & 0.23 \\
\hline \multirow[t]{2}{*}{ Glutamine } & Meningitis $(n=16)$ & 30.2 & 12.5 & 20.0 & 11.2 \\
\hline & Control $(n=7)$ & 33.1 & 12.7 & 20.9 & 5.6 \\
\hline \multirow[t]{2}{*}{ Serine } & Meningitis $(n=21)$ & 4.4 & 7.8 & 2.9 & 1.9 \\
\hline & Control $(n=9)$ & 2.6 & 1.1 & 3.1 & 1.7 \\
\hline \multirow[t]{2}{*}{ Glycine } & Meningitis $(n=16)$ & 3.6 & 1.5 & 4.0 & 2.7 \\
\hline & Control $(n=7)$ & 3.7 & 2.6 & 3.0 & 1.4 \\
\hline \multirow[t]{2}{*}{ Threonine } & Meningitis $(n=16)$ & 4.7 & 2.2 & 3.6 & 1.7 \\
\hline & Control $(n=6)$ & 4.9 & 2.3 & 3.9 & 1.2 \\
\hline \multirow[t]{2}{*}{ Taurine } & Meningitis $(n=9)$ & 2.7 & 1.6 & 3.5 & 2.9 \\
\hline & Control $(n=6)$ & 1.3 & 1.7 & 1.6 & 1.5 \\
\hline \multirow[t]{2}{*}{ Alanine } & Meningitis $(n=9)$ & 1.4 & 0.75 & $7.0 \dagger$ & 3.9 \\
\hline & Control $(n=6)$ & 1.7 & 1.0 & 2.3 & 1.7 \\
\hline
\end{tabular}

$* n$, number of animals.

$\dagger p<0.05$ (Wilcoxon signed-ranked test).

normalized relative to baseline values for each animal, glutamate increased by a mean of $470 \%$ in cortex during infection $(p<$ 0.05 ), with a wide variability between animals. Alanine also increased significantly at $22 \mathrm{~h}$ (Table 2 ). Alanine is considered to be a marker for anaerobic glycolysis (24), and interstitial alanine and lactate concentrations correlated well at $22 \mathrm{~h}$ of infection $(r=0.73, p<0.02)$. Furthermore, alanine and glutamate concentrations also correlated $(r=0.69, p<0.005)$. No other amino acids showed significant increases, but glutamine decreased by about $30 \%$ both in infected and control animals. Microdialysis concentrations of glutamate collected within 60 min after killing the animals confirmed that the probes were functioning with a massive increase of glutamate concentration to $16.0 \pm 18.27 \mu \mathrm{M}$.

\section{DISCUSSION}

This study was designed to compare concentrations of amino acids in the CSF and brain interstitial fluid during experimental bacterial meningitis. Interest in amino acids is stimulated by an attempt to understand the pathogenesis of brain injury during meningitis and the fact that EAA can cause direct neuronal injury when present at increased concentrations in the brain $(8$, $25,26)$. The role of this mechanism of CNS injury has not been explored in bacterial meningitis.

We found significant increases of glutamate, aspartate, glycine, taurine, and alanine concentrations in the CSF of infected animals. Glutamate and aspartate represent major EAA, whereas glycine and taurine play a role as inhibitory amino acids (27). The CSF concentrations of two amino acids that do not have a documented neurotransmitter function (serine and threonine) did not change during infection. These findings extend previous studies that have documented increased CSF concentrations of glutamate, aspartate, glycine, taurine, and alanine in patients with bacterial meningitis $(18,20)$. Increased concentrations of other individual amino acids, as well as of the total concentration of amino acids, have also been observed in patients with meningitis $(17,19,28)$. One study documented that patients with high CSF concentrations of amino acids had an increased incidence of death or neurologic sequelae, but no correlation was found in that study between amino acid concentrations and CSF leukocyte, lactate, or protein concentrations in the relatively small number of patients studied (28).

The mechanisms responsible for increased concentration of amino acids in the CSF during bacterial meningitis are not entirely clear. Disruption of the blood-brain barrier is a prominent early pathophysiologic change in meningitis and could possibly account for the increased CSF amino acid concentrations by passive diffusion from serum (29). This may, however, not be the only factor for at least two reasons. Concentrations of amino acids can be higher in the CSF than in serum during meningitis, indicating an additional intracranial source, and previous studies have not found a good correlation between CSF amino acid concentrations and CSF protein concentrations, which represent a good marker for blood-brain barrier disruption $(19,28,30)$. Increases in CSF protein concentrations have been well documented in our model of meningitis, but we have not measured CSF protein concentrations in the present study. Another possibility that has not been explored in detail is that the infection alters clearance from the CSF at different rates for different amino acids. With regard to intracranial sources of amino acids, release from cells recruited to the CSF as part of the inflammatory response or release from brain tissue in response to injury could account for the increase in CSF amino acid concentrations. In other models of CNS injury, tissue concentrations of amino acids have been found to be elevated in response to noxious stimuli such as ischemia and trauma $(1,4$, 5). It is noteworthy that two amino acids without recognized neurotransmitter function did not increase in CSF during meningitis in our study, which supports the brain as a source of the increased amino acid concentrations in the CSF. Cells in CSF could also contribute to the increased amino acid concentrations in CSF, but this has not been studied in sufficient detail.

Using the technique of microdialysis allowed us to measure interstitial fluid concentrations of amino acids as the disease evolved. This technique has previously measured increased interstitial concentrations of amino acids as well as other molecules in several animal models of brain injury $(1,3,4)$. Among the amino acids that increased significantly in CSF during meningitis, glutamate and alanine also showed increased interstitial concentrations in infected animals. Interpretation of the microdialysis results has to take into account the following points. First, while the interstitial amino acid concentrations appear low compared with CSF, the measured absolute concentrations in dialysate fluid are considerably lower than the true interstitial fluid concentrations because they reflect the limited extraction by the probes. Second, the extraction rate of the probes tends to decrease during use and thus leads to an underestimate of the relative increase in tissue concentrations over time. 
We observed a considerable variability of amino acid concentrations in interstitial fluid at the end of the infection that was not observed in control animals or at baseline, suggesting that this variability reflected the biology rather than being inherent to the technical procedure. For glutamate, the largest increase was $2020 \%$, and approximately $50 \%$ of the infected rabbits had concentrations above $200 \%$ of baseline, whereas the other animals showed minimal changes. This variability likely indicates that the pathologic processes in the brain that lead to increased amino acid concentrations are heterogeneous. This is supported by observations in a rat model of pneumococcal meningitis (21), where we found pronounced focality of the inflammation throughout the cortex of infected animals with evidence of associated, focal neuronal injury, findings that were confirmed in a few rabbits in the present study (unpublished observation). Similar focal changes have been described in a rabbit model of meningococcal meningitis (31) and in pathologic-anatomical studies in humans (32).

The mechanisms responsible for increased amino acid concentrations in brain interstitial fluid have not been identified. Ischemia is associated with a marked release of EEA and other amino acids from injured neurons $(7,26)$. We have previously shown that global cerebral blood flow is not markedly reduced in our model of meningitis, as long as animals do not develop hypotension $(15,16)$, and, early in the disease, blood flow is actually increased (13). Shock and global cerebral hypoperfusion were therefore probably not responsible for the changes in amino acid concentrations in this study. This is in contrast to a recent study published in abstract form (33), in which experimental Escherichia coli meningitis appeared to lead to increases in cerebral glutamate concentrations in response to profound septic shock developing as a result of the infection. Our studies do not exclude, however, that focal inflammation of the cerebral vasculature, as documented by our study in rats with pneumococcal meningitis (21), may lead to local cerebral ischemia that could account for the heterogenous release of glutamate. It remains to be determined whether other pathologic processes occurring during meningitis can lead to the release of amino acids from brain tissue.

Several conclusions regarding brain injury during bacterial meningitis can be drawn based on the findings of this study. First, the integrity of brain tissue is disrupted by the disease process sufficiently to lead to increased release of certain amino acids in brain interstitial fluid. The heterogeneity of this process may explain the variability observed in the amino acid measurements. Second, release from injured brain tissue appears to be in part responsible for the increased amino acid concentrations observed in the CSF during meningitis, but other components, such as increased blood-brain barrier permeability, altered clearance, and release from cells in the CSF, may also contribute. Finally, the increased concentrations of interstitial glutamate concentrations in infected animals suggest that excitotoxic neuronal injury may play a role in this disease.

\section{REFERENCES}

1. Benveniste H, Drejer J, Schousboe A, Diemer N 1984 Elevation of extracellular concentration of glutamate and aspartate in rat hippocampus during transient cerebral ischemia monitored by intracerebral microdialysis. J Neurochem 43:1369-1374

2. Butcher SP, Sanberg M, Hagberg H, Hamberger A 1987 Cellular origins of endogenous amino acids released into the extracellular fluid of the striatum during severe insulin-induced hypoglycemia. J Neurochem 47:722-728

3. Graham S, Shiraishi K, Panter S, Simon R, Fader A 1990 Changes in extracellular amino acid neurotransmitters produced by focal cerebral ischemia. Neurosci Lett 110:121-130

4. Globus MYT, Busto R, Dietrich WD, Martinez E, Valdez I, Ginsberg MD
1988 Effect of ischemia on in vivo release of striatal dopamine, glutamate and aminobutyric acid studied by intracerebral microdyalisis. J Neurochem $51: 1455-1464$

5. Faden AI, Demediuk P, Panter SS, Vink R 1989 The role of excitatory amino acids and NMDA receptors in traumatic brain injury. Science 244:798-800

6. Panter S, Yum S, Faden A 1990 Alteration in extracellular amino acids after traumatic spinal cord injury. Ann Neurol 27:96-99

7. Simon R 1989 Role of endogenous excitatory amino acid neurotransmitters in the pathogenesis of acute brain injury. Pediatr Infect Dis J 8:913-915

8. Choi DW 1988 Gluamate neurotoxicity and disease of the nervous system. Neuron 1:623-634

9. Moodley M, Bullock MR 1985 Severe neurological sequelae of childhood bacterial meningitis. S Afr J Med 68:566-570

10. Schwartz MN 1984 Bacterial meningitis: more involved than the meninges. $N$ Engl J Med 311:912-914

11. Sande MA, Täuber MG, Scheld WM, McCracken GHJ 1989 Pathophysiology of bacterial meningitis. Summary of the workshop. Pediatr Infect Dis J 8:929-933

12. Tunkel AR, Wispelwey B, Scheld WM 1990 Bacterial meningitis: recent advances in pathophysiology and treatment. Ann Intern Med 112:610-623

13. Pfister HW, Koedel U, Haberl RL, Dirnagl U, Feiden W, Kuckdesche LG, Einhaupl KM 1990 Microvascular changes during the early phase of experimental bacterial meningitis. J Cereb Blood Flow Metab 10:914-922

14. Paulson OB, Brodersen P, Hansen EL, Kristensen HS 1974 Regional cerebral blood flow, cerebral metabolic rate of oxygen, and cerebrospinal fluid acidbase variables in patients with acute meningitis and with acute encephalitis. Acta Med Scand 196:191-198

15. Tureen JH, Täuber MG, Sande MA 1992 Effect of hydration status on cerebral blood flow and cerebrospinal fluid lactic acidosis in rabbits with experimental meningitis. J Clin Invest 89:947-953

16. Täuber MG, Burroughs M, Niemöller UM, Kuster H, Borschberg U, Tuomanen E 1991 Differences of pathophysiology in experimental meningitis caused by three strains of Streptococcus pneumoniae. J Infect Dis 163:806811

17. Buryakova A, Cand M, Stynsky I 1975 Amino acid composition of cerebrospinal fluid in acute neuroinfections in children. Arch Neurol 32:28-31

18. Heiblim D, Evans H, Glass L, Agbayani M 1978 Amino acid concentrations in cerebrospinal fluid. Arch Neurol 35:765-768

19. Corston R, McGale E, Stonier C, Hutchinson E, Aber G 1979 Abnormalities of cerebrospinal fluid amino-acids in purulent meningitis. J Neurol Neurosurg Psychiatr 42:881-886

20. San Joaquin V, Khai N, Seaale T, Rennert O 1982 Increased cerebrospinal fluid-free amino acid concentrations in children with bacterial meningitis. Scand Infect Dis 14:23-26

21. Täuber MG, Kennedy SL, Tureen JH, Lowenstein DH 1992 Experimental pneumococcal meningitis causes central nervous system pathology without inducing the $72 \mathrm{kDa}$ heat shock protein. Am J Pathol 141:53-60

22. Dacey RG, Sande MA 1974 Effect of probenecid on cerebrospinal fluid concentrations of penicillin and cephalosporin derivatives. Antimicrob Agents Chemother 6:437-441

23. Guerra-Romero L, Täuber MG, Founier MA, Tureen JH 1992 Lactate and glucose concentrations in brain interstitial fluid, cerebrospinal fluid, and serum during experimental pneumococcal meningitis. $J$ Infect Dis 166:546550

24. Nilsson G, Lutz P 1991 Release of inhibitory neurotransmitters in response to anoxia in turtle brain. Am J Physiol 261:R32-R37

25. Kohmura E, Yamada K, Hayakawa T, Kinoshita A, Matsumoto K, Mogami H 1990 Hippocampal neurons become more vulnerable to glutamate after subcritical hypoxia: an in vitro study. J Cereb Blood Flow Metab 10:877884

26. Rothman SM, OIney JW 1986 Glutamate and the pathophysiology of hypoxicischemic brain damage. Ann Neurol 19:105-111

27. McGeer P, McGeer E 1989 Amino acid neurotransmitters. In: Siegel GJ, Agronoff B, Alberts RW, Molinoff P (eds) Basic Neurochemistry: Molecular, Cellular, and Medical Aspects. Raven, New York, pp 311-332

28. Briem H, Hultman E, Kalin M, Lundberg P 1982 Increased total concentration of amino acids in the cerebrospinal fluid of patients with purulent meningitis. J Infect Dis 145:346-350

29. Quagliarello VJ, Long WJ, Scheld WM 1986 Morphologic alterations of the blood-brain barrier with experimental meningitis in the rat. J Clin Invest 77:1085-1095

30. Davson H, Welch K, Segal M 1987 Physiology and Pathophysiology of the Cerebrospinal Fluid. Churchill Livingstone, Edinburgh, pp 269-301

31. Branham SE, Lillie RD 1932 Observations on experimental meningitis in rabbits. Public Health Rep 47:2137-2150

32. Cairns H, Russell DS 1946 Cerebral arteritis and phlebitis in pneumococal meningitis. J Pathol Bacteriol 58:649-665

33. Perry V, Young R, During M, Aquilla W 1991 Is excessive glutamate a cause of brain injury in acute bacterial meningitis? An in vivo cerebral microdialysis study. Ann Neurol 29:991(abstr) 